\title{
Development of a new bench for puncturing of irradiated fuel rods in STAR hot laboratory
}

\author{
B. Petitprez, P. Silvestre, P. Valenza, A. Boulore (Fuel Research Department, CEA Cadarache), T. \\ David (Sealing Laboratory Maestral, CEA Marcoule)
}

\begin{abstract}
A new device for puncturing of irradiated fuel rods in commercial power plants has been designed by Fuel Research Department of CEA Cadarache in order to provide experimental data of high precision on fuel pins with various designs. It will replace the current set-up that has been used since 1998 in hot cell 2 of STAR facility with more than 200 rod puncturing experiments. Based on this consistent experimental feedback, the heavy-duty technique of rod perforation by clad punching has been preserved for the new bench. The method of double expansion of rod gases is also retained since it allows upgrading the confidence interval of volumetric results obtained from rod puncturing. Furthermore, many evolutions have been introduced in the new design in order to improve its reliability, to make the maintenance easier by remote handling and to reduce experimental uncertainties.
\end{abstract}

Tightness components have been studied with Sealing Laboratory Maestral at Pierrelatte so as to make them able to work under mixed pressure conditions (from vacuum at $10^{-5}$ mbar up to pressure at 50 bars) and to lengthen their lifetime under permanent gamma irradiation in hot cell. Bench ergonomics has been optimized to make its operating by remote handling easier and to secure the critical phases of a puncturing experiment. A high pressure gas line equipped with high precision pressure sensors out of cell can be connected to the bench in cell for calibration purposes. Uncertainty analyses using Monte Carlo calculations have been performed in order to optimize capacity of the different volumes of the apparatus according to volumetric characteristics of the rod to be punctured. At last this device is composed of independent modules which allow puncturing fuel pins out of different geometries (PWR, BWR, VVER).

After leak tests of the device and remote handling simulation in a mock-up cell, several punctures of calibrated specimens have been performed in 2016. The bench will be implemented soon in hot cell 2 of STAR facility for final qualification tests. PWR rod punctures are already planned for 2018.

Index Terms - fuel rod, pressure, puncturing, remotehandling, experimental uncertainties

\section{OBJECTIVES OF THE NEW ROD PUNCTURING BENCH}

Fission gas release from fuel under irradiation and the resulting pressure increase are key parameters influencing the behaviour of a nuclear Pressurized Water Reactor (PWR) fuel rod in nominal and accident conditions. This data is obtained experimentally at the CEA Cadarache centre by a device that has been used in hot cell 2 of the STAR facility since 1998 with more than 200 PWR rod puncturing experiments [1]. In order to broaden the CEA's experimental capability, a new device for puncturing of irradiated fuel rods from different types of commercial power plants has been designed by the CEA Fuel Research Department at Cadarache. Based on the consistent experimental feedback obtained with the current device, the double expansion puncturing method [2] has been kept for the new device. The design of this new device has been optimised to simplify operation and maintenance by remote-handling, to guarantee reliable puncturing experiments and to reduce uncertainties associated with the experimental results.

\section{ASSEMBLING, OPERATION AND MAINTENANCE OF THE BENCH BY REMOTE-HANDLING}

In compliance with the ALARA approach, all the phases of the device service life have to be performed in the hot cell by remote-handling. At the same time, availability of the bench has to be guaranteed to allow up to twenty rod puncturing experiments per year. Thus, a modular design has been adopted with several independent components to be assembled directly in the hot cell. As for the current machine, three main volumes - the calibration assembly (VB), the rod assembly (VM) and the expansion assembly (VD) - comprise the gas circuit of the new bench as shown in Figure 1.

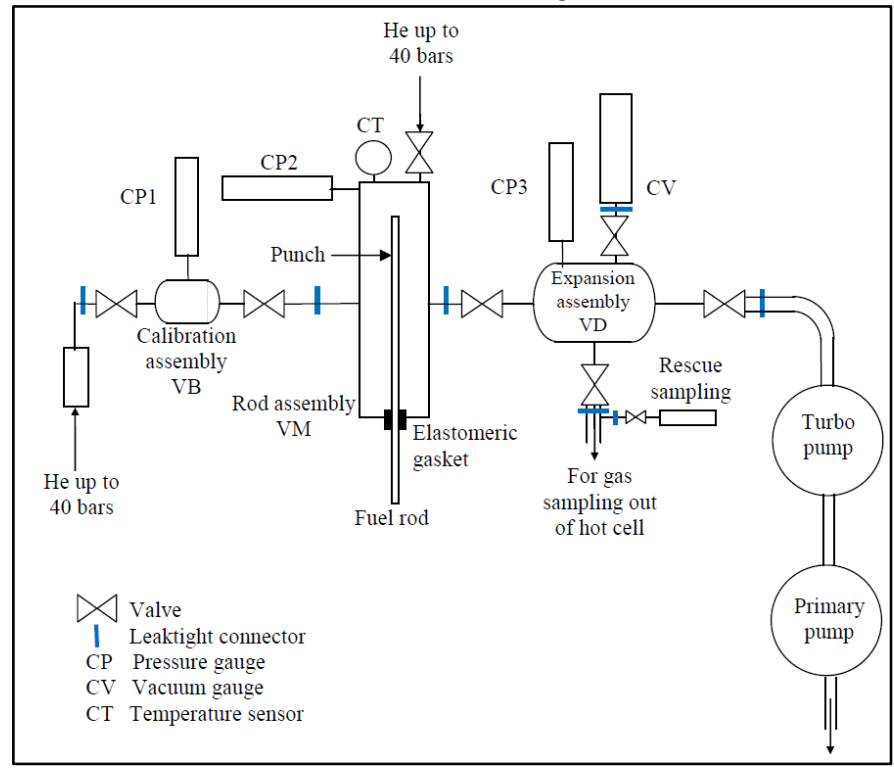

Fig. 1. Schematic diagram of the puncturing device 
The three volumes can then be connected and disconnected independently thanks to gasket fittings easy to handle with remote manipulators. A guiding support receives the different volumes and helps to connect them to each other with alignment pins and fasteners. At the end of assembling, a punching column is fixed on the rod assembly and the support. Pressure gauges have also been designed to be screwed onto the modules by remote-handling through an appropriate replaceable sealing joint as represented in Figure 2.

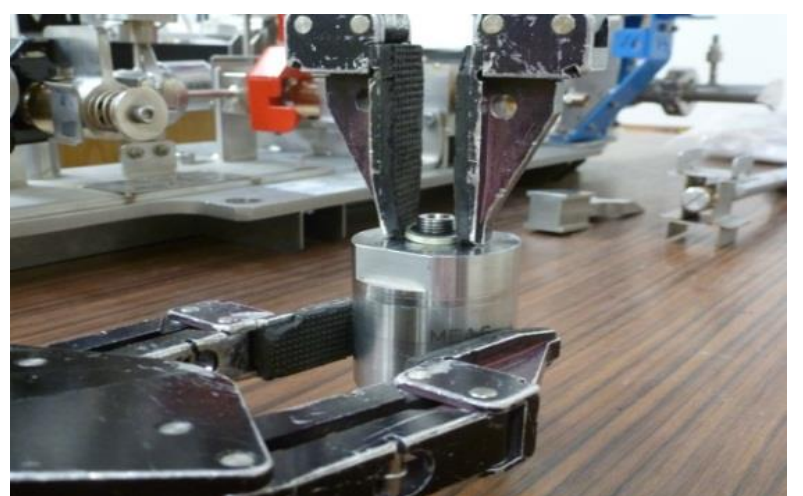

Fig. 2. Pressure gauge seal replacement by remote-handling

Leak tightness is key parameter of the puncturing device; its leak rate should remain below $10^{-7} \mathrm{~Pa} \cdot \mathrm{m}^{3} / \mathrm{s}$ for all operating conditions, i.e. from $10^{-3} \mathrm{~Pa}$ (secondary vacuum) to $5.10^{6} \mathrm{~Pa}$ of helium. As metallic seals could not be used in this case, it was crucial to find an elastomeric seal material resistant enough to permanent gamma irradiation in a hot cell and suitable for our working conditions.

This is why different kinds of elastomers were tested in the MAESTRAL Laboratory [3] at the CEA Pierrelatte centre to characterise leak-tightness and mechanical behaviour after several months of gamma irradiation in hot cell. Figure 3 shows the leak-tight behaviour during a 3-phase pressure test which reproduces a puncturing experiment for two different elastomers at three stages of irradiation.
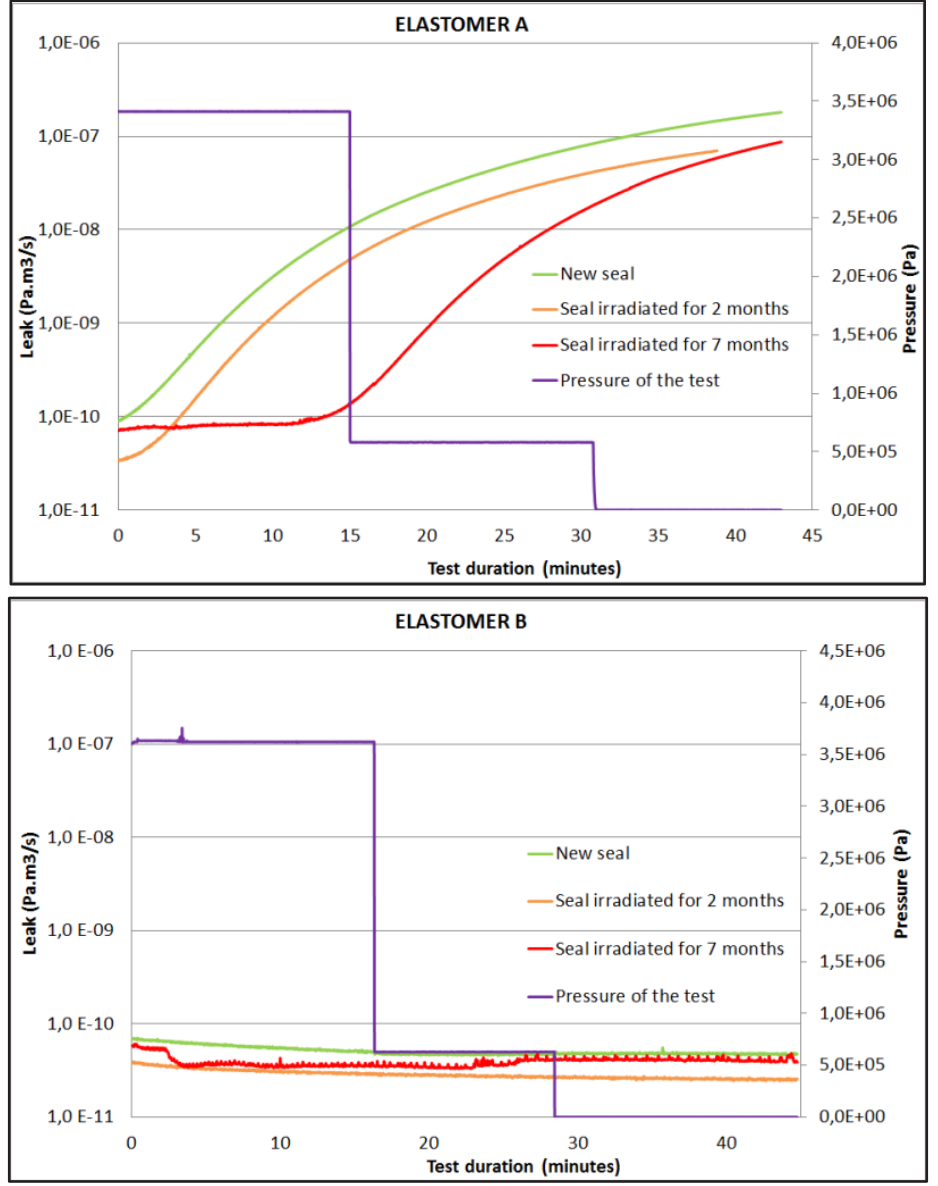

Fig. 3. Comparison of leak-tight behaviour between two different elastomers (fresh and after irradiation)

The most appropriate elastomer was chosen as the sealing material to be used for the fittings, the gauge seals and the gasket around the fuel rod. Additional tests in the MAESTRAL Laboratory made it possible to determine the optimised torque for tightening the different seals of the bench using a torque wrench adapted for remote-handling. The tests performed in the MAESTRAL Laboratory also allowed defining the replacement frequency of the device sealing components.

The bench ergonomics were also studied to simplify operations with remote manipulators and to make it easier to monitor the actions trough the shielded window. For instance, all of the device valves have been identified and designed for secure handling as shown in Figure 4.

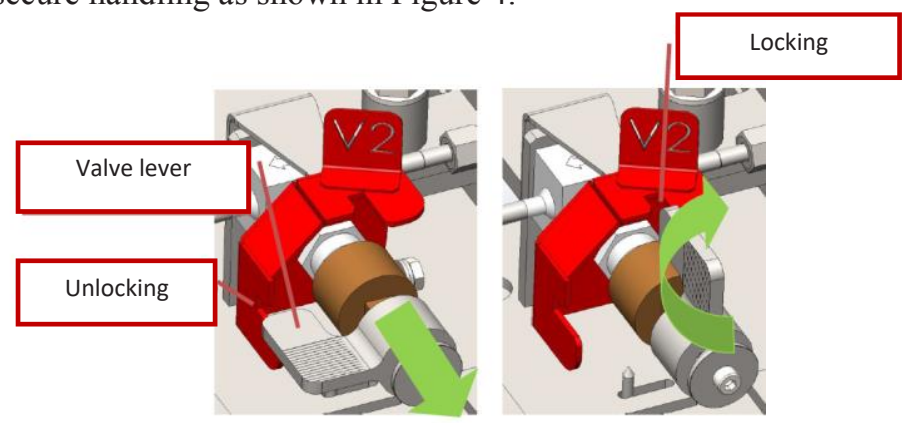

Fig. 4. Bench valves design for remote-handling 


\section{A MORE RELIABLE PUNCTURING BENCH FOR ALL TYPES OF FUEL ROD GEOMETRIES}

As rod puncturing is a one-shot experiment, the leaktightness of the puncturing device has to be guaranteed once the fuel rod has been placed in rod assembly for the whole experiment, from rod punching to gas sampling. This is why a helium pressurization line capable of providing up to $5.10^{6} \mathrm{~Pa}$ of helium has been implemented in hot cell 2 of the STAR facility. Thanks to a pressure connection on the puncturing device rod assembly (see Figure 1), the line can be connected directly to the rod assembly while the fuel rod is in place ready to be punched. It is therefore possible to perform a leak test in the rod assembly at the pressure level expected after rod puncturing. This change in the experimental procedure with the new device secures the puncturing experiment.

The reliability of the puncturing experiment is also guaranteed by the double expansion method [2], which is an extension of the conventional single expansion method with an additional experimental step. Using the double expansion puncturing method with the new bench sketched in Figure 1 enables to express rod inner pressure Pc and rod free volume $\mathrm{VL}$ as a system of two equations with two unknowns:

$$
\left\{\begin{array}{l}
\mathrm{Pc} * \mathrm{VL}=\mathrm{P} 1 *(\mathrm{VL}+\mathrm{VM}) \\
\mathrm{P} 1 *(\mathrm{VL}+\mathrm{VM})=\mathrm{P} 2 *(\mathrm{VL}+\mathrm{VM}+\mathrm{VD})
\end{array}\right.
$$

where P1 is the pressure measurement after the first expansion of rod gases in VM and P2 is the pressure measurement after the second expansion in VD.

The rod free volume is still measured by single expansion at the end of the puncturing experiment to confirm the measurement and it can be compared with the result obtained by double expansion. It can also be used as a back-up measurement. After this stage of the experimental procedure, the volumetric data of the fuel rod has been determined and confirmed, i.e. the rod free volume, the inner rod pressure at $20^{\circ} \mathrm{C}$ and the total gas volume in the rod at $20^{\circ} \mathrm{C}$ and 1 atmosphere.

It is then necessary to determine the elementary and isotopic compositions of the gas contained in the fuel rod, in particular to assess the fission gases released from fuel since the beginning of irradiation. To do this, it is necessary to collect a leak-tight sample of the rod gas that has expanded in the puncturing device circuit. The sample is then sent to the mass spectrometry laboratory for analysis. All these operations require many handling operations in hot cell and later in a glove box outside the hot cell. In order to prevent the risk of gas loss during this phase, a removable sampling phial (see Figure 5) has been added to the new device. In case of gas loss during sampling, the phial can be used as back-up for gas sampling. After decontamination, the phial can be removed from the cell so the gas content can be transferred into a secondary ampoule to be sent for mass spectrometry analysis.

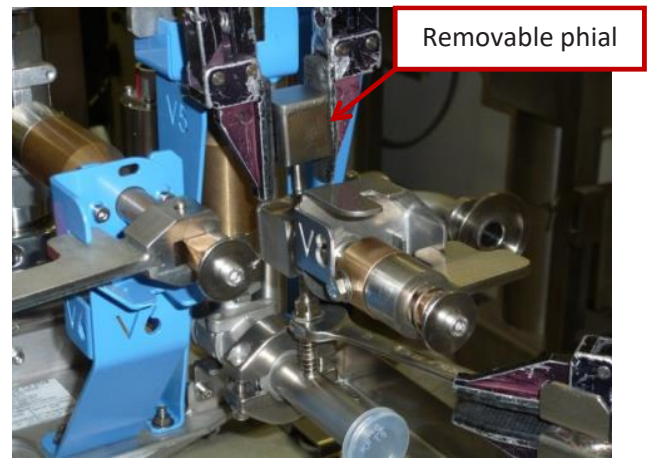

Fig. 5. Back-up gas sampling system for the new puncturing bench

The modular design of the new puncturing bench allows receiving all geometries of commercial fuel rods irradiated in nuclear plants. A rod assembling VM such as that represented in Figure 6 can be designed and built on demand for any given geometrical specifications (outer diameter and plenum length of fuel rod). It can then be installed on the bench and connected to the two other modules (calibration volume VB and expansion volume VD) by means of the gasket fittings. In the same manner, a pressure gauge can be screwed into the CP2 position of the new rod assembly. At last two different positions are available on the punching column for the failing mass that allows adapting the perforation energy to the cladding characteristics of the rod to be punctured.

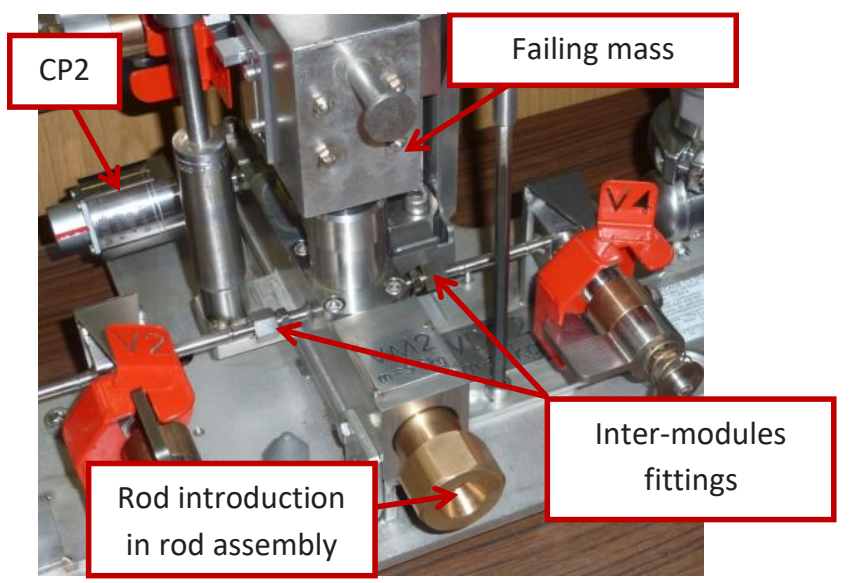

Fig. 6. Rod assembly design for the new puncturing bench

\section{PROVIDING MORE ACCURATE EXPERIMENTAL RESULTS}

During any reactor cycle, the plant operator has to take into account pressure margins in fuel assemblies to maintain a certain level of flexibility for power incursions and/or burn-up increase [4]. This monitoring uses fuel performance codes that have been qualified based on the data-base of rod puncturing results. The rod pressure margin calculations also take into account experimental uncertainties on these puncturing results. This is why it is essential to reduce as much as possible the experimental uncertainties associated to the quantities of interest (rod inner pressure, total gas volume in the rod, free volume) obtained with the new bench.

An optimisation approach using Monte-Carlo calculations was adopted in the early design phase to determine the 
dimensions of the sampling volume VB, the rod assembly VM and the expansion volume VD. First the URANIE software developed by the CEA has been used for uncertainty quantification [5], sensitivity analysis and optimisation to implement the measurement model defined by the mathematical relations between all quantities known to be involved in a rod puncturing experiment. Then the optimisation problem consisted in identifying the input data to fix (e.g. volumetric characteristics of the rod to puncture and measuring features of the three pressure gauges) and the quantities to make vary within a given range (VB, VM, VD and $\mathrm{P} 1$ corresponding to the pressure measurement after the first expansion). Lastly Monte-Carlo calculations were performed with varying set combinations (P1; VB; VM; VD) and the results were filtered to minimise the standard deviation results. Figure 7 schematises the optimisation process for the given input data with all Monte-Carlo calculations represented in green lines and the resulting standard deviation histogram for rod pressure calculations $(\mathrm{stdPc})$. The blue lines represent the combinations of values $(\mathrm{P} 1 ; \mathrm{VB}$; VM; VD) that minimise the value of stdPc.

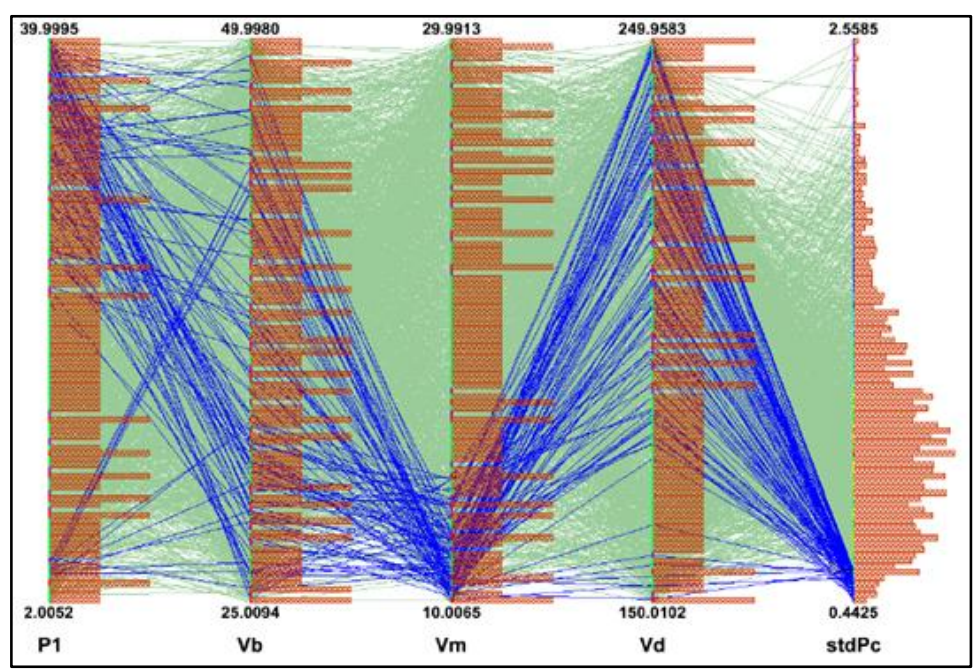

Fig. 7. Optimization process to design bench volumes (VB, VM, VD) that minimise rod pressure uncertainty (StdPc)

From a practical point of view, these combinations allow defining capacity ranges for VB, VM and VD where puncturing result uncertainties are minima for a given rod. Specifications for the new puncturing bench were finally established according to the results of these optimisation calculations.

The accuracy of the on-line pressure and temperature measurements also contributes to the resulting uncertainties related to the experimental rod puncturing results. Therefore the helium pressurisation line has been equipped with high precision pressure gauges located out of cell to take into account the instrumentation drift on the three pressure gauges implemented on the puncturing bench (CP1, CP2 and $\mathrm{CP} 3$ ). These high precision sensors measure the absolute pressure with a maximal relative error of $\pm 0.01 \%$ and are periodically calibrated at the French metrology laboratory LNE. They can then be used as secondary measurement standards to calibrate the pressure gauges $\mathrm{CP} 1, \mathrm{CP} 2$ and $\mathrm{CP} 3$ directly in the hot cell providing an operating pressure measurement accuracy better than $\pm 0.5 \%(\mathrm{k}=2)$. Furthermore a new calibration procedure has been developed to determine the capacity of the puncturing bench reference volumes VB and VD with an accuracy close to $\pm 1 \%(\mathrm{k}=2)$. These calibrations provide thus the standard measurement uncertainties $\mathrm{u}(\mathrm{xi})$ associated with the different parameters $\mathrm{xi}$ that are used in the uncertainty budget of a puncturing experiment. According to uncertainty propagation methodology by second order Taylor development, the following equation illustrates the calculation used to determine the uncertainty associated with the inner rod pressure result Pc obtained at the end of rod puncturing experiment:

$u\left(P_{c}\right)=\sqrt{\sum_{i}\left(\frac{\partial P_{c}}{\partial C P i}\right)^{2 *} u(C P i)^{2}+\left(\frac{\partial P_{c}}{\partial V B}\right)^{2 *} u(V B)^{2}+\left(\frac{\partial P_{c}}{\partial V D}\right)^{2 *} u(V D)^{2}+\left(\frac{\partial P_{c}}{\partial T}\right)^{2 *} u(T)^{2}}$

where $\mathrm{CPi}$ correspond to pressure measurements by $\mathrm{CP} 1, \mathrm{CP} 2$ and CP3 during the experiment and $\mathrm{T}$ is the rod gas temperature measured during the experiment.

The final uncertainty $\mathrm{U}(\mathrm{Pc})$ on the rod pressure result is : $\mathrm{U}(\mathrm{Pc})=\mathrm{k}^{*} \mathrm{u}(\mathrm{Pc})$ with $\mathrm{k}=2$ so as to guarantee a coverage interval at $95 \%$. It is important to note that $\mathrm{u}(\mathrm{Pc})$ is the analytical expression corresponding to the standard deviation $\operatorname{std}(\mathrm{Pc})$ obtained by probabilistic calculations used for the volume bench design. So comparison of results obtained by both methods is an efficient way of validating the experimental uncertainty estimations.

\section{CONCLUSION}

Many improvements have been introduced in the design of the new rod puncturing device and the puncturing experiment procedure has been upgraded as well. As a result, the bench maintenance can be performed entirely by remote-handling within a few days and it will now take two days to complete a puncturing experiment. Furthermore the bench has been designed to puncture all types of commercial fuel rod geometries. Its reliability has been improved for evaluating the quantities of interest (rod inner pressure, total volume of gas in rod, free volume) as well as for assessing the rod gas composition. Presently, work has be done to improve the accuracy of the puncturing results so the relative uncertainties can be reduced to $\pm 4 \%(\mathrm{k}=2)$ for the rod inner pressure and $\pm 5 \%(\mathrm{k}=2)$ for the rod free volume. The new rod puncturing bench will be implemented in hot cell of STAR facility in 2018 and it should be fully qualified at the end of the year. PWR rod punctures are already planned afterwards.

\section{ACKNOWLEDGMENTS}

The authors would like to thank AREVA-NP and EDF for their financial and technical support in this study.

\section{REFERENCES}

[1] Irradiated Fuel Puncturing Devices Implemented at the LECA-STAR Laboratory of CEA-Cadarache, Y. Pontillon, B. Gleizes, H. Capdevilla, P. Silvestre, P. Plume, $45^{\text {th }}$ HOTLAB conference, 2008 
[2] A new apparatus for determination of the free volume of a fuel rod using the double expansion method, L. Desgranges, M.H. Faure, A. Thouroude, Nuclear Technology, vol. 149, January 2005

[3] http://technetics.com/engineering/rd-innovation/

[4] Nuclear fuel safety criteria technical review (second edition), ISBN 978-92-64-99178-1, OECD 2012

[5] URANIE: The CEA/DEN Uncertainty and Sensitivity platform, $F$. Gaudier, Procedia Social and Behavioral Sciences 2 (2010) 7660-7661 